\title{
DISTRIBUTION OF NANOPARTICLES IN THE PREGNANT RAT: THE MORPHOLOGIC AND SPECTROSCOPIC STUDY
}

\author{
Vytautas Kulvietis ${ }^{1,2}$, Violeta Žalgevičiene $\dot{e}^{3}$, Janina Didžiapetriene ${ }^{1}$, \\ Danutè Bulotiene ${ }^{1}$, Ričardas Rotomskis ${ }^{1,2}$ \\ ${ }^{1}$ Biomedical Physics Laboratory, the Institute of Oncology, \\ Vilnius University, Lithuania \\ ${ }^{2}$ Biophotonics Group of the Laser Research Center, Faculty of Physics, \\ Vilnius University, Lithuania \\ ${ }^{3}$ Faculty of Medicine, Vilnius University, Lithuania
}

\begin{abstract}
The nanoparticles (NP) applications in industry and biomedicine are growing despite of superficial understanding about the mechanisms of NP biological interactions and the possible toxicity. The beneficial NP applications in medicine, as well as potential adverse effects, first of all depend on the NP accumulation and localization in the organism. The human in pre-natal stages is more sensitive to toxic materials than the adult organism and, therefore, it is physiologically protected from harmful agents by the selective transport across the placental barrier. However, there is a lack of studies on the NP penetration through this barrier and the accumulation in foetus.

In this study fluorescence spectroscopy and confocal microscopy methods were employed to investigate the distribution of polyethylene glycol (PEG) coated semiconductor quantum dots (QD) in the pregnant rat model. The main results indicate that QD are systemically distributed in the body and can be found in all the investigated organs, including placenta and uterus $3 \mathrm{~h}$ after the intraperitoneal injection. However, QD could not be detected in the foetal tissues (embryo, yolk sac placenta, umbilical cord). The study shows that QD mainly accumulate in the maternal blood sinuses of the placenta and the QD passage to the foetus is prevented by the placental barrier.
\end{abstract}

Key words: nanoparticles, fluorescence, imaging, placenta, barrier. 


\section{INTRODUCTION}

Nanoparticles (NP) are organic and inorganic substances in the size range of 1-100 $\mathrm{nm}$ and humans have been exposed to such particles throughout their history. However, the industrial era dramatically changed sources, doses, and the types of NP. Nanotechnology is a rapidly developing field leading to an increase of engineered NP with conceptually new physical and chemical properties, which might induce novel effects in biological systems $[6,7]$. The number of commercial NP-based products in food, cosmetics and medicine is expanding [1, 15]. This phenomena raises concerns about NP accumulation, long-term retention in organism and the forthcoming toxic effects.

Quantum dots (QD) are semiconductor NP in the size of 2-10 nm and they have superior optical properties when compared with organic dyes. QD have broad absorption spectra, bright photoluminescence (PL), they are photostable and can be easily chemically modified for biological functionalization. It was shown that QD can be toxic in vitro and in vivo and the adverse effects depends on QD physicochemical and environmental factors $[3,7,10]$. However, the biological interaction of NP mainly depend on their size and surface coating $[4,13]$ and these properties can be easily tuned in the case of QD. It makes QD a perfect model of NP to investigate the fundamental mechanisms of the NP biodistribution and physiological effects.

The studies of the NP penetration through the placental barrier are important because of few aspects. Firstly, is the evaluation of possible embryotoxicity and teratogenicity of nanoagents. Secondly, the NP which do not pass to the foetus could be used as the drug delivery platform during pregnancy to avoid the effect on embryogenesis [11]. Finally, the specialized NP which permeate the placental barrier could be used for embryo-targeted prenatal diagnostics and therapy. By now there are only few in vivo studies on the NP accumulation in foetus and there is a lack of knowledge of general patterns of the NP transport across the maternal-foetal barrier.

In this study we used the methods of fluorescence spectroscopy and confocal microscopy to investigate the accumulation of CdSe/ZnS PEG coated QD in the tissues of the pregnant rat and to evaluate their penetration across the placental barrier to the foetus. 


\section{MATERIALS AND METHODS}

\section{Animal breading}

Albino Wistar rats (9-11 weeks old) were obtained from the State Research Institute Center of Innovative Medicine (Vilnius, Lithuania). Animals were housed under the conditions of constant temperature, humidity and the standard light /dark cycle $(12 \mathrm{~h} / 12 \mathrm{~h})$. Food and fresh drinking water were available ad libitum. The study was approved by the Lithuanian Animal Care and Use Committee (No 0019; 2001-2005).

After being acclimated for at least 7 days, female rats were mated overnight with the males of the same strain. Vaginal smears from each female rat were collected and subjected to microscopic examination on the following morning in order to determine the oestrous cycle and the presence of sperm. The day of sperm detection in vaginal smears was designated as day 0 of gestation.

The CdSe/ZnS quantum dots coated with polyethylen glycol (PEG) were used for experiments (Qtracker-655, non-functionalized, Invitrogen Inc.). The stock solution was diluted up to $0.8 \mu \mathrm{M}$ in saline and injected intraperitoneally on the day 18 of gestation. The animals of the control group were injected with pure saline.

\section{QD pharmacokinetics measurements}

The $50 \mu$ l blood samples were punctuated from the tail vein before (control sample) and after the QD injection (t: $0.5-24 \mathrm{~h}$ ). In total 4 animals were used for the experiments. The blood was instantly mixed with $50 \mu \mathrm{l}$ heparin solution to prevent coagulation. The solution was diluted up to $1.5 \mathrm{ml}$ with saline and centrifuged for $10 \mathrm{~min}$ at $1500 \mathrm{rcf}$. The supernatant was used for fluorescence spectroscopy. The relative QD concentration in the blood plasma was assessed by subtracting the autofluorescence spectra and evaluating the PL intensity at the peak. The data from different experiments $(n=4)$ was normalized to the maximum intensity and averaged. The averages were fitted using the biphasic dose response model (1):

$y(x)=A_{1} /\left(1+10^{\wedge}\left(\left(b_{1}-x\right) * h_{1}\right)\right)+A_{2} /\left(1+10^{\wedge}\left(\left(b_{2}-x\right) * h_{2}\right)\right)$,

$\mathrm{x}$ - independent variable (time), $\mathrm{A}_{1,2}, \mathrm{~b}_{1,2}, \mathrm{~h}_{1,2}$ - fitting parameters. 


\section{Fluorescence spectroscopy}

$3 \mathrm{~h}$ after injection all the rats were subjected to the Caesarean section in the state of neuroleptoanalgesia (Calipsol $0.5 \mathrm{ml}$ per one rat) on day 18 [8]. The internal organs, including uterus with the formed embryos, were removed. The excised organs were washed in saline and drained.

The fluorescence spectra were measured using the Varian Cary Eclipse spectrometer coupled with the fiber optics module. The blood solution samples were measured using the standard $1 \mathrm{~cm}$ plastic cuvettes. The excitation light of $480 \mathrm{~nm}$ was used.

\section{Sample preparation and fluorescence microscopy}

The internal organs including uterus, placenta and embryo were sectioned using the cryomicrotome setup. The section slides were divided in two groups: untreated and stained with haematoxylin/eosin (HE). The analysis was performed using the Nikon Eclipse TE-2000 fluorescence microscope with $\mathrm{C} 1$ confocal scanning system (objectives x10/0.7 and x60/1.4 Plan Apo VC oil). The argon ion laser was used for the excitation light of $457 \mathrm{~nm}$ and $488 \mathrm{~nm}$.

\section{RESULTS}

\section{Fluorescence spectroscopy}

After the intaperitoneal injection, the QD presence in the blood plasma was observed after 15 minutes by the means of fluorescence microscopy. The QD concentration reached its maximum after $\sim 2.5 \mathrm{~h}$ and started to decrease afterwards (Figure 1.). After $24 \mathrm{~h}$ QD could not be detected in the blood plasma indicating their clearance from blood and/or degradation. The increase is associated to the QD absorption from the intraperitoneal space via the lymphatic or blood vessels. The QD decrease is thought to be mainly determined by the uptake of the reticuloendothelial system and accumulation in tissues [4]. 


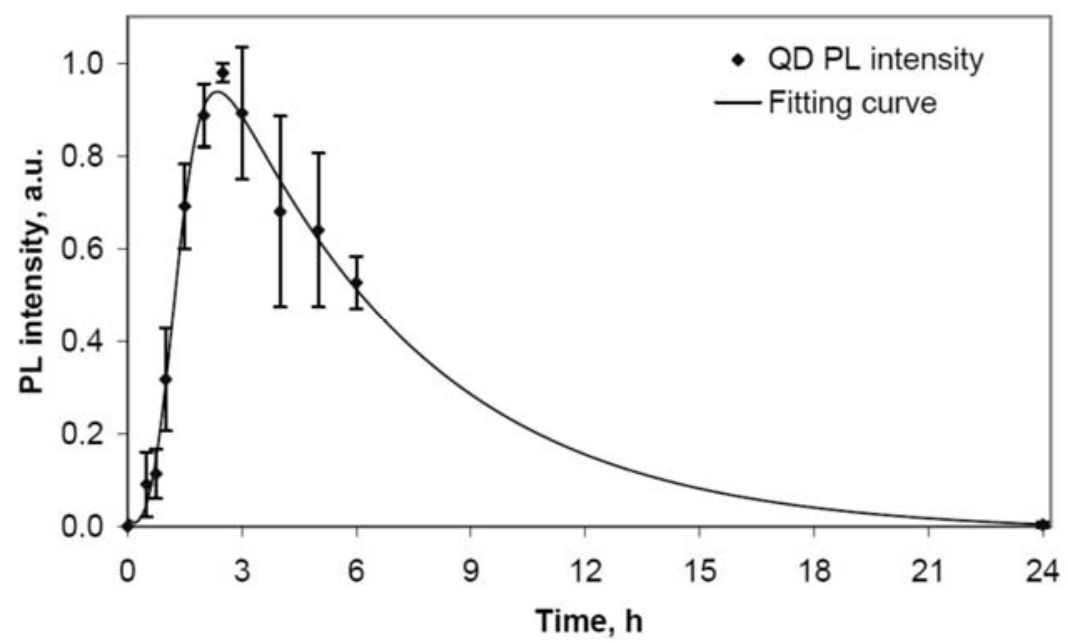

Figure 1. The kinetics of QD concentration in the blood plasma of the pregnant rat after the intraperitoneal injection. The data are represented as the mean \pm standard deviation from $n=4$ animals.

The QD incubation interval of $3 \mathrm{~h}$ hours was selected for the evaluation of QD distribution in tissues as it represents the maximum likelihood to detect QD by means of fluorescence techniques. QD accumulation in tissues was evaluated by means of fluorescence spectroscopy. The characteristic QD PL was seen in the spectra of all the investigated organs, including liver, lungs, muscle, heart, thymus, etc. These results indicate that QD were distributed systemically due to blood circulation.

QD could also be detected in the uterus and placenta tissues (Figure 2.). However QD PL was not registered in the embryonic tissues: embryo, yolk sac placenta and umbilical cord. The fluorescence of the yolk sac placenta surrounding the embryo is mainly addressed to the PL peak at $619 \mathrm{~nm}$ which is attributed to the endogenous porphyrins (Figure 3.). The porphyrin accumulation in the pregnant rat was observed earlier [5]. 


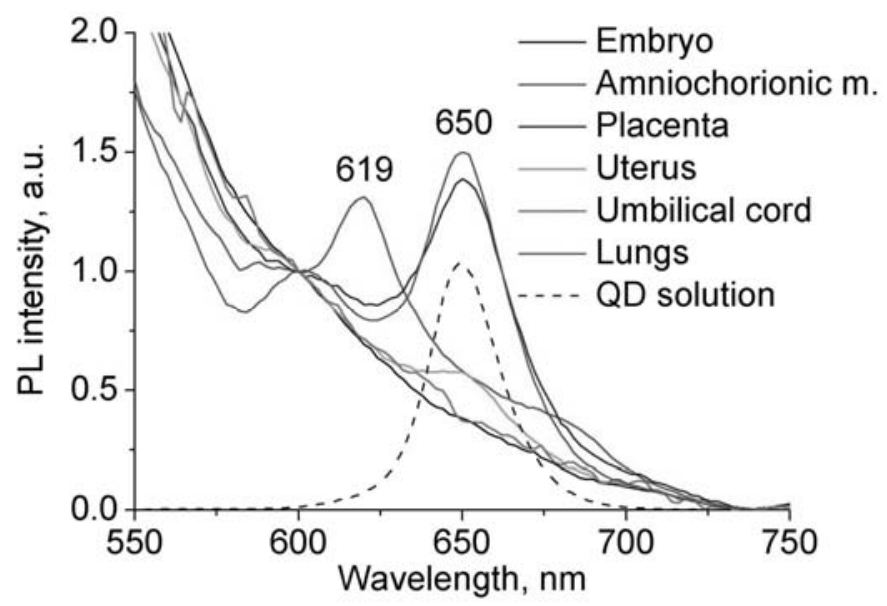

Figure 2. The fluorescence spectra of rat tissues indicating the presence of QD in the maternal tissues (uterus, placenta) $3 \mathrm{~h}$ after the QD injection.

\section{Microscopic analysis of tissue samples}

The cryotome dissection was used to prepare tissue slides for the microscopy analysis. The prepared samples were described by histological examination using the standard HE staining technique under low magnification (Figure 3). In the sample of placenta the characteristic structures of decidua, junctional zone and labyrinthine zone layers are easily identified.
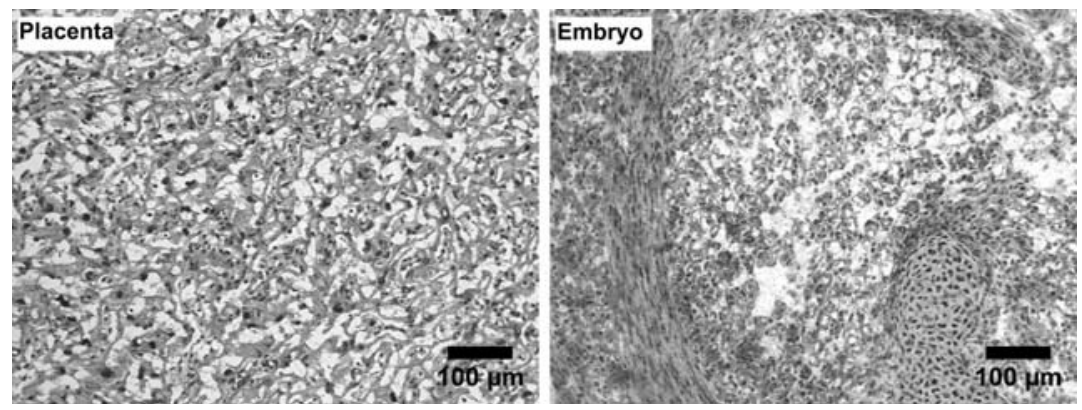

Figure 3. The H\&E stained cryosections of the placenta (the labyrinthine zone) and the embryo, objective x10/0,25. 
The unstained slides were used to investigate the QD localization. The confocal microscope coupled with a spectral detector enables the discrimination of the QD PL from the tissue autofluorescence with high spectral resolution. This technique revealed that QD are accumulated in the labyrinthine zone of the placenta (Figure 4). QD appeared distributed in the samples not homogenously, but patterned, indicating QD accumulation in the areas with a lower autofluorescence background (represented green). In the labyrinthine zone the maternal blood sinuses lack of endogenous fluorophores which are more abundant in the connective foetal tissue, therefore maternal blood results in lower fluorescence intensity and darker areas in the image when compared with foetal tissue. In this way, QD are mostly distributed in the maternal blood sinuses.
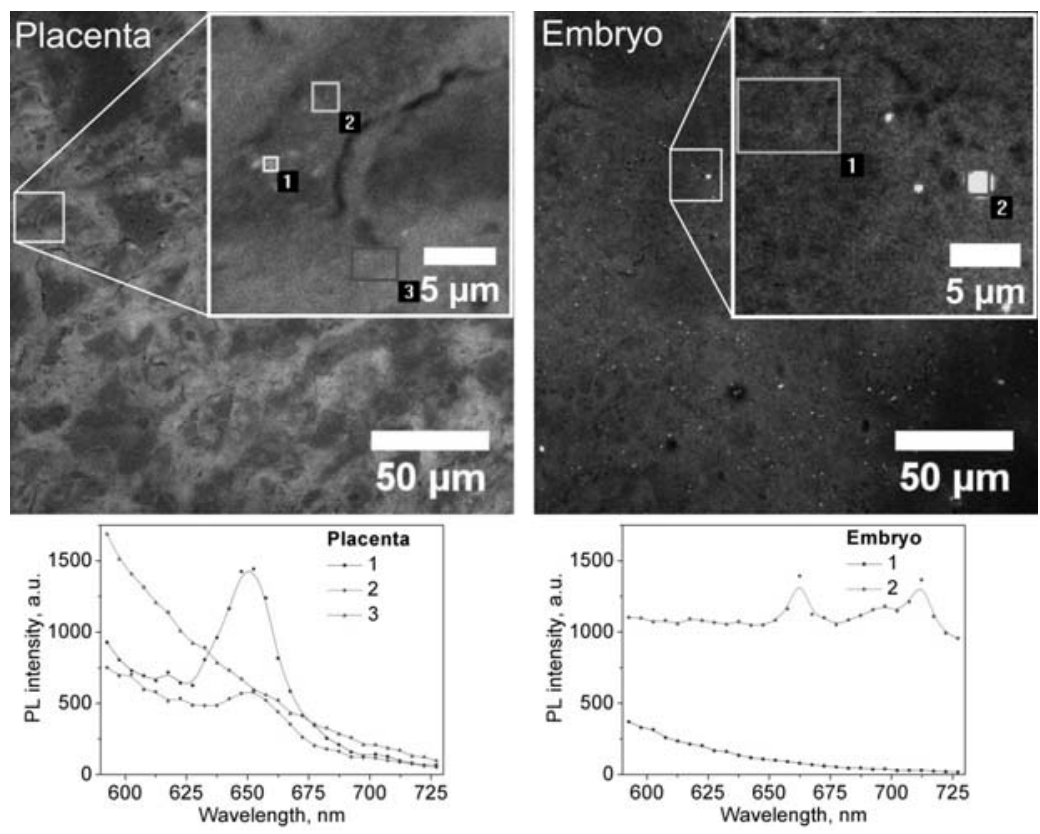

Figure 4. Confocal microscopy image of the rat placenta (labyrinthine zone) and the embryo tissue slides $3 \mathrm{~h}$ after QD injection. The patterned red distribution of QD PL (at $650 \mathrm{~nm}$ ) in the placenta is mainly attributed to the tn the maternal blood sinuses of the placenta. The characteristic QD PL was not registered in the embryo sample, objective x60/1,4. 
The examination of the embryo tissues showed no appearance of QD PL in the samples. Some yellowish fluorescent endogenous fluorophores were seen, but the PL spectra were distinct from the QD PL spectra found in the placenta. These results confirm the spectroscopic findings.

\section{DISCUSSION}

By now there are only few in vivo studies on nanoparticles (NP) penetration through the placental barrier and the forthcoming accumulation in the foetus. Recently $\mathrm{Chu} \mathrm{M}$ et al. investigated the transplacental transport of thiol-capped CdTe QD in mice [3]. Twentyfour hour after intravenous administration the highest QD accumulation was registered in liver and spleen, while the cadmium concentration in the foetus reached only $0.6 \%$ of the injected dose. The fluorescence microscopy technique was unable to detect QD in the embryonic tissues. The surface stabilization with polyethylene glycol (PEG) or $\mathrm{SiO}_{2}$ decreased QD uptake to the puppies. The embryotoxic effects of QD were found to be dependent on the cadmium dose in the foetus. However, the authors indicate that the performed mass spectrometry assay was not able to discriminate between QD and free cadmium ions, therefore it is not possible to say if QD penetrated the placental barrier or they were degraded in the maternal organism and the formed cadmium ions were transported to the foetus [3].

Another study reported pregnancy complications in mice induced by the silica and titanium dioxide $\left(\mathrm{TiO}_{2}\right)$ nanoparticles [15]. The wholebody optical imaging analysis showed the accumulation of fluorescently labelled $70 \mathrm{~nm}$ size silica and $143 \mathrm{~nm}$ size $\mathrm{TiO}_{2} \mathrm{NP}$ in placenta 24 hours after intravenous NP injection. However the most intense fluorescence for all NP was observed in the liver. Electron microscopy revealed that NP were localized in the trophoblast cells of the placenta, as well as in the foetal liver and foetal brain tissues. The quantitative analysis was not performed. However, the silica NP of bigger size $-300 \mathrm{~nm}$ or $1000 \mathrm{~nm}$ - were not observed in the placenta or foetuses. The NP induced adverse effects on the embryo development such as growth inhibition, resorptions, placental dysfunction and other functional changes. The observed physiological changes were related to the NP penetration across the barrier and they were not induced using the $300 \mathrm{~nm}$ and 1000 nm NP [15]. 
The lack of in vivo studies on NP transplacental passage and the disambiguous results of different groups raise the need for additional investigations on physiological NP effects to embryogenesis. Our results show that fluorescence spectroscopy and confocal microscopy methods can be used to investigate the accumulation and pharmacokinetics of fluorescent nanoparticles in the experimental animals. The main findings indicate that 3 hours after intraperitoneal QD injection they are systemically distributed throughout the organism with blood circulation and can be found in all the organs, including the uterus and the placenta. High QD concentration in blood resulted in the QD appearance in the maternal sinuses of the placenta. However, they were not detected in the foetal tissues using fluorescence spectroscopy and microscopy techniques. It shows that the QD penetration across the placental barrier is highly limited. According to ex vivo studies, the dendrimers appear in the foetal compartment already $15 \mathrm{~min}$ after the NP addition to the maternal perfusate [11] and their concentration keeps rising up to $6 \mathrm{~h}$. The concentration of polystyrene NP in the foetal compartment was saturated after $3 \mathrm{~h}$ of perfusion [14]. These data indicate that the period of $3 \mathrm{~h}$ used in our experiments should be sufficient for QD to penetrate across the placental barrier.

It was also shown that the PEG coated gold NP accumulated in the placenta without passing to the foetal tissue [12]. Different ex vivo studies on human models showed that the NP penetration through the placental barrier highly depends on the size and the surface coating of the NP $[11,12,14]$. We used PEG coated QD which increases the overall size of the nanoparticles and minimizes the interactions with biomolecules. The increase in size reduces the passive passage of NP through the biological barriers [2] and the NP interactions with proteins are essential for NP cellular adhesion and the forthcoming endocytosis by the trophoblast cells $[9,13]$. Therefore the PEG coating could result in the lower QD transplacental passage.

It is worth mentioning, that the QD PL intensity remained stable during fluorescence imaging which is not characteristic of the conventional organic dyes used for fluorescence microscopy. The effect of photo bleaching is one of the main limitations in fluorescence-based methods and it can be overcome using the semiconductor QD.

For conclusion, PEG coated $\mathrm{CdSe} / \mathrm{ZnS}$ quantum dots distributed systemically in the body of the pregnant rat $3 \mathrm{~h}$ after the intraperitoneal 
injection and accumulated in all the investigated organs, including the uterus and the placenta. Fluorescence microscopy revealed that QD mainly accumulate in the maternal blood sinuses of the placenta, but they were not found in the foetal tissues. The fluorescence-based investigations show that the NP passage to the foetus is prevented by the placental barrier. Further studies using the particles of different sizes and materials are naturally needed in order to conclusively evaluate the mechanisms of the NP transplacental transport and to extract the knowledge on possible adverse or beneficial effects of the NP application in medicine.

\section{ACKNOWLEDGEMENTS}

This research was funded by the grant (No. MIP-10440) of the Research Council of Lithuania and by the grant (No. 2004-LT0036-IP-1NOR) of Norwegian financial mechanism.

\section{REFERENCES}

1. Adair B.M. (2009) Nanoparticle vaccines against respiratory viruses. Wiley Interdiscip Rev Nanomed Nanobiotechnol, 1, 4, 405-414.

2. Choi H.S., Liu W., Misra P., Tanaka E., Zimmer J.P., Itty Ipe B., Bawendi M.G., Frangioni J.V. (2007) Renal clearance of quantum dots. Nat Biotechnol, 25, 10, 1165-1170.

3. Chu M., Wu Q., Yang H., Yuan R., Hou S., Yang Y., Zou Y., Xu S., Xu K., Ji A., Sheng L. (2010) Transfer of quantum dots from pregnant mice to pups across the placental barrier. Small, 6, 5, 670-678.

4. Delehanty J.B., Mattoussi H., Medintz I.L. (2009) Delivering quantum dots into cells: strategies, progress and remaining issues. Anal Bioanal Chem, 393, 4, 1091-1105.

5. Grazeliene G., Zalgeviciene V., Didziapetriene J., Zukiene J., Sukackaite A., Legenis V., Rotomskis R. (2006) Accumulation of photosensitizer in rat embryos (a spectroscopic study). Medicina (Kaunas), $42,2,142-146$.

6. Gupta R.C. (2007) Veterinary toxicology. USA, Academic Press Elsevier, 2007. $1201 \mathrm{p}$.

7. Hardman R. (2006) A toxicologic review of quantum dots: toxicity depends on physicochemical and environmental factors. Environ Health Perspect, 114, 2, 165-172. 
8. Hayes A.W. (1994) Principles and Methods of Toxicology. New York, Raven Press, 1006-1036.

9. Kelf T.A., Sreenivasan V.K.A., Sun J., Kim E.J., Goldys E.M., Zvyagin A.V. (2010) Non-specific cellular uptake of surface-functionalized quantum dots. Nanotechnology, 21, 285105.

10. Lin C.H., Chang L.W., Chang H., Yang M.H., Yang C.S., Lai W.H., Chang W.H., Lin P (2009). The chemical fate of the $\mathrm{Cd} / \mathrm{Se} / \mathrm{Te}-\mathrm{based}$ quantum dot 705 in the biological system: toxicity implications. Nanotechnology, 20, 21, 215101.

11. Menjoge A.R., Rinderknecht A.L., Navath R.S., Faridnia M., Kim C.J., Romero R., Miller R.K., Kannan R.M. (2011) Transfer of PAMAM dendrimers across human placenta: Prospects of its use as drug carrier during pregnancy. J Control Release, 150, 3, 326-338.

12. Myllynen P.K., Loughran M.J., Howard C.V., Sormunen R., Walsh A.A., Vähäkangas K.H. (2008) Kinetics of gold nanoparticles in the human placenta. Reprod Toxicol, 26, 2, 130-137.

13. Tan S.J., Jana N.R., Gao S., Patra P.K., Ying J.Y. (2010) SurfaceLigand-Dependent Cellular Interaction, Subcellular Localization, and Cytotoxicity of Polymer-Coated Quantum Dots. Chem Mater, 22, 7, 2239-2247.

14. Wick P., Malek A., Manser P., Meili D., Maeder-Althaus X., Diener L., Diener P.A., Zisch A., Krug H.F., von Mandach U. (2010) Barrier capacity of human placenta for nanosized materials. Environ. Health Perspect, 118, 3, 432-436.

15. Yamashita K., Yoshioka Y., Higashisaka K., Mimura K., Morishita Y., Nozaki M., Yoshida T., Ogura T., Nabeshi H., Nagano K., Abe Y., Kamada H., Monobe Y., Imazawa T., Aoshima H., Shishido K., Kawai Y., Mayumi T., Tsunoda S., Itoh N., Yoshikawa T., Yanagihara I., Saito S., Tsutsumi Y. (2011) Silica and titanium dioxide nanoparticles cause pregnancy complications in mice. Nat. Nanotechnol, 6, 5, 321-328.

\section{Address for correspondence:}

Vytautas Kulvietis

Institute of Oncology, Vilnius University

104 room, Biomedical Physics Laboratory

P. Baublio 3b, Vilnius LT-08406, Lithuania

E-mail: vytautas.kulvietis@vuoi.lt 